\title{
Preface to the second edition
}

Photochemistry and photocatalysis in organic synthesis have advanced at a fast pace over the past five years. From a rediscovered methodology in chemistry, the use of light in synthesis emerged in the first 15 years of this century into an enabling technology improving many important reactions, such as cross-coupling reactions or cycloaddition. In addition, new reaction modes were established, which were previously difficult to achieve or even unknown, such as the formation of nitrogen atom centered radicals of amides, alpha amino radical formation under mild conditions or various $\mathrm{C}-\mathrm{H}$ activation steps.

In the last years, nearly all areas of organic synthesis have seen new applications of light as a reagent to drive transformations. Photocatalysis evolved into a standard technique in synthesis and is widely applied in academic and industry laboratories around the world. First examples of photocatalytic production of molecules on industrial scale have been reported and the number of such applications is expected to rise. After the "gold rush" of fast discoveries of many photocatalytic reactions, detailed spectroscopic investigations start to unravel the mechanistic details. However, the field still has a long way to go to reach a comprehensive understanding of reaction mechanisms at the molecular level.

The second edition of this book presents updated chapters and three new chapters summarizing the background and recent achievements of the photochemistry of radical ions, decarboxylative reactions and biomimetic photooxidations.

The research presented in this book has been supported by generous funding from the Deutsche Forschungsgemeinschaft, which established from 2010 to 2019 a graduate research training group on Chemical Photocatalysis (GRK 1626) at the University of Regensburg in collaboration with groups at the universities of Munich (TUM, LMU) and Leipzig.

Regensburg, Munich, December 2019 Burkhard König, Thorsten Bach 
